GALAXIES IN SMALL GROUPS 
THE PHOTOGRAPHY OF GROUPS OF GALAXIES

B. A. Vorontsov-Velyaminov

Sternberg Astronomical Institute, Moscow University, U.S.S.R.

\section{IMPROVEMENTS IN PHOTOGRAPHY}

The method of combining several negatives together is at last coming into use. Forty years ago this method was used for the first time by Leontovsky in Leningrad. Putting together many negatives he was able to do photometry of M31 to fainter magnitudes than was possible photoelectrically. The method of preliminary baking of emulsions is more effective when it is done in a nitrogen atmosphere and still better in hydrogen. Spectacular results with the last method were obtained by A. Smith (1977). With a $70 \mathrm{~m}$ exposure on IIIa-J plates there are no traces of the planetary nebula the Helix. After hydronization the same exposure reveals the overexposed image of this object. Arp and Lorre (1976) obtained striking results on IIIa-J emulsions using the process of deconvolution which improves the negatives. By means of some kind of filtration, removing faint stars and those of medium brightness etc., they improved the resolution of the extended images and obtained a better contrast. The authors published their photographs of the Stefan group and of the jet in M87. As a result of deconvolution it becomes evidence that NGC 7320 is much nearer to us than its apparent neighbouring galaxies because it is clearly resolved into HII regions and star clusters.

One has to be extremely cautious about the detection of faint details on negatives which have suffered strong intensification. The procedure also intensifies spurious details. Can you imagine what would have happened if Arp and Lorre forgot to warn the reader that the dark rings around all condensations in the jet of M87 are but the result of their processing?

\section{THE APPLICATION OF PHOTOGRAPHY TO GROUPS}

The methods of colorimetry and photographic photometry through colour filters, which enable one to discern stellar populations and the distribution of ionized hydrogen has been used very seldom for groups. 
The evaluation of their distances from the apparent diameters of HII regions can be used. Unfortunately the total dispersion of their linear diameters is very much greater than the value found by Sandage and Tammann if larger volumes of the Metagalaxy and interacting galaxies are considered.

In determining distances to the Stefan group, Arp used an average value for the diameters of HII regions of $200 \mathrm{pc}$ for all its members. However Vorontsov et a1. (1974) have drawn attention to the fact that in the interacting pair NGC 2535-6 the largest HII regions exceed 2000 pc in length. These measurements were made on a photograph taken by Arp in the light of $\mathrm{HB}$ and we adopted the cosmological redshift which Arp himself adopted for this object. Our paper, which discussed the Stephan group, was never published. The referee rejected its publication advancing arguments of the kind: "the authors apparently mistook for HII regions in this galaxy the images of the bright field stars far from NGC 2535" and so on.

It would be desirable to evaluate the masses of the members of groups from their luminosities and morphological types. However even the integral magnitudes and the colours in most cases are only known very crudely or are unknown altogether. Estimates of morphological types are often uncertain due to the peculiarity of the objects or to overexposure. It is most difficult to differentiate apparently spheroidal objects.

The problem of membership of groups is more complicated. The most recent studies show that apparent proximity alone does not guarantee a physical relationship. The criterion formerly considered to be best the similarity of redshifts - suffers from the lack of red shifts. But even this criterion is violated by $\operatorname{Dr}$ Arp because he believes in very large non cosmological red shifts and he gives us no optical criteria for deciding which objects must have non cosmological red shifts (excluding QSS).

In the group VV 166 one apparent member has red shift $1000 \mathrm{~km} \mathrm{~s}^{-1}$ smaller than that of the other members. Kormendy and Sargent (1974) argue that it is a chance projection because it shows no interaction with its spiral neighbour. But this argument is not convincing because this elliptical galaxy can only respond feebly to tidal forces. The E galaxy NGC 71, an undisputable member of this group, does not show deformations due to its neighbours.

The sole optical peculiarity of E galaxies was our finding (Vorontsov et al. 1962) that some giant E galaxies ( $\mathrm{gE}$ ), some of them multiple, some in clusters and some outside, have large coronae and are best denoted by $\mathrm{gEH}, \mathrm{H}$ meaning Halo or Haze. Their discovery has been ascribed falsely and persistently in the West to W. Morgan. He denoted them by $\mathrm{cD}$ only three years later. Besides it introduces confusion, since by D he also means "disk galaxies". 
The discovery of interacting galaxies in 1958 drew attention to the importance of taking photographs, even on a small scale, with long exposures, in order to study these enigmatic bridges and tails. The Palomar Sky Atlas made it possible to identify, describe and measure nearly 2000 such objects in the MCG (Morphological Catalogue of Galaxies). The discovery of extradense groups - nests of galaxies indicates the next task: to obtain photographs which might discern the individual peculiar and irregular objects the images of which have coalesced or are in contact. This requires large scale and exposures with a light ratio $1: 4$ of 4 to 1 minute to resolve the number of nuclei. This has not been done so far, but in this way a peculiar object can transform under our eyes into several galaxies. We are convinced that such a break down in fact happens.

Having little time, I will show a few slides to illustrate how a series of exposures presents "cuts" at various levels of a galaxy. In my Atlas (Vorontsov 4 and 5) of interacting galaxies I stress that from the study of overexposed images of the Palomar Sky Atlas I can only suspect that a given object is not single, but is a tight system. Rephotography of such objects by $\operatorname{Dr} \operatorname{Arp}$ (1966) and by the observers at the 6-m telescope confirmed this suspicion in most cases. There still exists the uncertainty introduced by the chance projections of foreground stars and of very compact galaxies. I will show first some nests, then chains (a particular case of nests) and cases where the structure of the objects is enigmatic, let alone their origin. Below are shown some of the VV objects, discovered in 1958-1964, and recently photographed with the 6-m telescope by I. D. Karachentsev, M. F. Shabanov and J. P. Korvjakovsky. For permission to show them I also thank cordially the Director of SAO Dr I. M. Kopylov.

VV 644. In compiling the MCG in 1961, I described this object as two galaxies in a close interaction, nearly coalescent. The 6-m photograph by Dr Karachentsev shows that in fact it is a nest of two pairs of compact galaxies. Both consist of a compact elongated galaxy in contact with a compact elliptical. A 5 th member of this nest is suspected. No bridges or tails are present. Markarian put this "object" on his list of blue galaxies under catalogue number 8 . Only this made D. Weedman measure its properties: $U-B=-0.52$ and absolute magnitude $\mathrm{M}_{\mathrm{V}}=-21$. The components have $\mathrm{M}_{\mathrm{V}}$ from -19 to -18 .

VV 556. A nest of two groups. The first consists of a minichain ( 3 members in contact). The other consists of a large diffuse object including a spheroidal galaxy and a bridge to a galaxy with a tail.

VV 568. This seems to be a nest of galaxies in a common envelope. There are two bright $\mathrm{E}$ and three faint IrI galaxies. Objects of such appearance are called "clumpy irregulars" by Heidmann and Casini. The irregular IrI galaxies have no such compact condensations. We expect that nests of dwarf irregular galaxies must be more frequent at the present late stage of evolution of the Metagalaxy. They represent a late stage of fragmentation of galaxies. 
VV 600. This is a complicated mixture of arms and filaments. One spiral arm is seen edgewise next to small galaxies connected by a filament. In addition there are 7 faint, nearly starlike objects. The three brightest of them probably form a chance projection of a triple star, but this must be checked spectrosopically.

VV16 = NGC 5996-94. At first glace, it might be considered to be a single SB galaxy, but... To the right of the bar there is an object akin to a nest of 5 dwarf but compact galaxies in contact, possibly far away. Note the peculiar structure of the bar ( 3 parts detached), the great asymmetry of the matter in the arms and a possible connection with the smaller early spiral to the left. Is it not all the late stage of fragmentation of a single system?

VV 470. This is not a minichain, but a triple galaxy of "twice M51" type. The central object is a spiral galaxy seen nearly edgewise. Near its plane it is connected with two symmetrical and symmetrically situated companions, each with a strong tail. The ratio of masses here could enable one to construct a tidal theory akin to the one so ingeniously compiled for M51 by the Toomres, separately for every companion. However the simultaneous close encounter of two similar companions moving in different planes with nearly parabolic velocities is most improbable. The improbability is of much higher order than that of a chance configuration of sprayed grains. Of 160 systems of M51 type which we have found, over ten belong to the "twice M51" type. Some of the components at the end of spiral arms are dwarfs of the $\operatorname{IrI}$ type, or large HII regions. In two cases their location on a single arm was spectroscopically recognized by Arp (1970).

VV 20. In this system a pair of $E(?)$ galaxies has a faint tail to the 1 eft, possibly produced by tides. In addition a very strong spiral bridge stretches from them to the big E-galaxy below. We find in it 3-4 condensations - a minichain in a common envelope. But we stress the impossibility of extracting so massive a formation from these tiny galaxies. Besides the latter are also connected to the big E-galaxy by a straight bridge. In the case of VV 19 too, as well as connections by means of two arms, the galaxies are connected by a straight bridge as is found in the cases of M51 and the others. Such bridges are not predicted by the tidal theory.

VV $523=$ NGC $3991=$ Haro 5. On the overexposed photograph in the PSA I mistook this object for a "cudgel" - a single body. Proper exposures showed that this blue object is a multimembered chain. Six

Page opposite: The pictures present enlargements to 2 arc sec per mm of the photographs of some interacting galaxies obtained at the prime focus of the $6-\mathrm{m}$ telescope. North is at the top, West to the right; otherwise, they are shown by arrows. The numbers correspond to the VV numbers of the objects. For details, see text. 


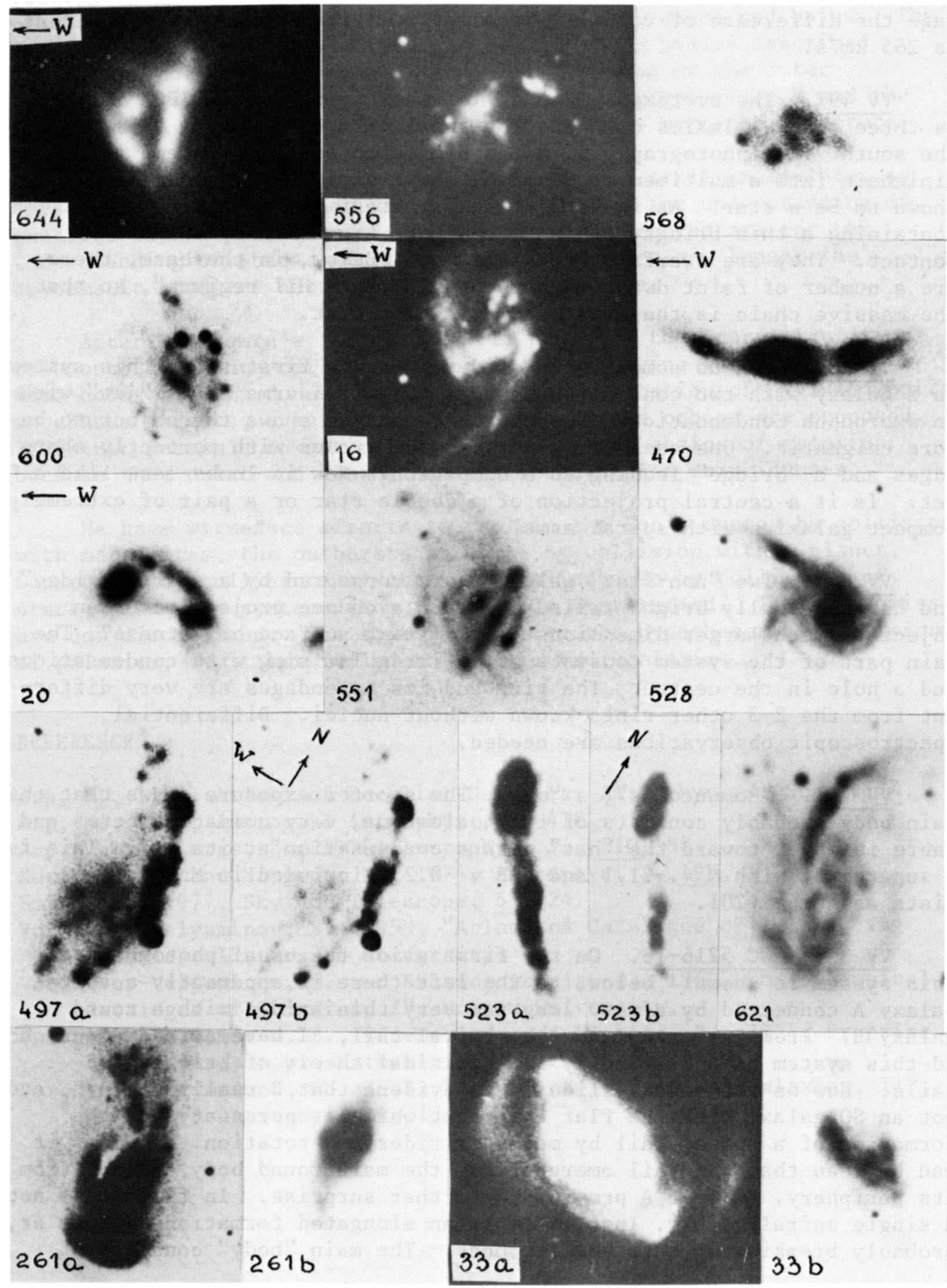


of the galaxies are in contact and the largest is somewhat apart, but is connected to the rest. In total $M=-21$, the members having $M_{V}=-19$ and less. They are compact with strong emission lines. According to Page the difference of velocity between the largest member and the rest is $265 \mathrm{~km} / \mathrm{s}$.

VV 497. The overexposed image of this system on the PSA appears as three round galaxies connected by short filaments with a blue haze to the south. The photography with the 6-m telescope transforemd this minichain into a multimembered chain, while one of the members was shown to be a star. As in VV 523 the brightest member, after a gap containing a thin bridge, begins a chain of 4 or more members in contact. They are dwarfs of the IrI type. Below, in the haze, there are a number of faint dwarf members or "isolated HII regions", so that the massive chain is the main part of a blue nest.

VV 528. Let us compare two slides. On the first slide this system is a galaxy with two concentric systems of spiral arms and a "jet" with an amorphous condensation. The shorter exposure shows the object to be more enigmatic. One sees two superimposed circles with perfectly sharp edges and a "bridge" leading to a companion. Now it looks less like a jet. Is it a central projection of a double star or a pair of extremely compact galaxies with spiral arms?

VV 621. Two "non-flat" galaxies are connected by a short "bridge" and have unusually bright tails. Is this a chance projection on an object of much larger dimensions but of faint surface brightness? The main part of the system consists of an irregular ring with condensations and $a$ hole in the centre. The ring and its appendages are very different from the 2-3 other rings known without nuclei. Differential spectroscopic observations are needed.

VV 261. "Homunculus", a "man". The shorter exposure shows that the main body probably consists of two coalescent, very compact spheres and there is a jet toward the "hat" with a condensation at its end. This is a supergiant with $\mathrm{M}=-21.1$ and $\mathrm{U}-\mathrm{B}=-0.25$, included in Markarian's lists as number 201 .

VV $33=$ NGC 5216-18. On the first slide the usual photograph of this system is shown: below, to the left there is apparently a spiral galaxy A connected by a very long and very thin bridge with a round galaxy B. From B emerges a bright spiral tail. I have already mentioned this system as a difficulty for the tidal theory of bridges and tails. Now on the second slide it is evident that $B$ really is an $E$, and not an S0 galaxy with the flat component which is necessary for the formation of a spiral tail by means of tides and rotation. Besides it can be seen that the tail emerges from the main round body and not from its periphery. Object A provides a further surprise. In fact it is not a single spiral galaxy. Instead it is an elongated formation, irregular, probably breaking up into smaller ones. The main "body" consists of 
3-4 coalescent compact galaxies. They form an excellent nest. The nest is in contact with the elongated body. From the point of contact two spiral arms emerge from the nest. To the right above there is a nebulous condensation and two faint "Brackets" - above and below. The long bridge toward galaxy $B$ emerges not from the bodies described above, and not from the spiral arms, but from one of the outer "brackets". The very complicated and amazing structure of B and the details of A cannot be accounted for by a purely mechanical picture.

Our knowledge of the nature of galaxies is still incomplete, as these examples show. They are as important for the understanding of galaxies as the phenomena of New stars and Supernovae are for the understanding of stars. It is a shame to neglect any longer the systematic study of nests and chains of galaxies.

After Dr Toomre's lecture it will be shown that our calculations rule out the chance collisional origin of pure ring galaxies. The same holds true in connection with the interpretation of dividing pairs as chance mergers, even if very eccentric closed orbits are supposed. It is not possible to set aside tight nests and chains of galaxies because they exist and cannot be considered chance coincidences.

We have witnessed efforts to explain the Sun's energy by collisions with meteorites, the outbursts of Novae by collision with a planet, Supernovae by collisions of stars, spiral nebulae by collisions of stars, planetary systems from the spirals thus formed. These theories are now discredited. The same fate awaits the hypotheses of collision of galaxies, as it has failed to explain radiogalaxies.

\section{REFERENCES}

Arp, H., 1966, Atlas of Peculiar Galaxies, Astrophys. J. Supp1. 14. Arp, H., 1970, Astron. \& Astrophys., 3, 418.

Arp, H. and Lorre, Z., 1976, Astrophys. J., 210, 58.

Kormendy, J. and Sargent, W., 1974, Astrophys.J.,193, 19.

Smith, A., 1977, Sky and Telescope, 53, 24.

Vorontsov-Velyaminov, B., 1959, "At $1 \overline{\text { as }}$ and Catalogue of Interacting Galaxies, I", Moscow University.

Vorontsov-Velyaminov, B. and Krasnogorskaja, A., 1962, "Morphologica1 Catalogue of Galaxies, I", Moscow University.

Vorontsov-Velyaminov, B., Efremov, J. and Komberg, B., 1974, Preprint of the Inst. App1. Mathematics, Moscow.

Vorontsov-Velyaminov, B., 1977, "Atlas of Interacting Galaxies, II", Astron. \& Astrophys. Supp1., 28, 1. 


\section{DISCUSSION}

Heidmann: I would like to emphasise what Dr Vorontsov-Vel'yaminov said about the importance of measuring radial velocities for his nests in order to find their absolute magnitudes. According to their intrinsic luminosities, they may be either classical resolved irregular galaxies, or giant irregulars of the clumpy type pointed out last year by Casini and myself or tight groups, i.e. nests, of galaxies. 